\title{
Rhizophyllum LINDSTRÖM, 1866 (RUGOSA) DEL DEVÓNICO INFERIOR (LOCHKOVIENSE) EN EL SE DE LAS MONTAÑAS DE TIAN-SHAN (NO DE CHINA)
}

\author{
Francisco SOTO' y Weihua LIAO \\ ' Departamento de Geología, Universidad de Oviedo, 33005 Oviedo. \\ 2 Nanjing Institute of Geology and Palaeontology, Academia Sinica. \\ Chi-Ming-Ssu, 210008 , China.
}

Soto, F. y Liao, W. 1998. Rhizophyllum Lindström, 1866 (Rugosa) del Devónico Inferior (Lochkoviense) en el SE de las Montañas de Tian-Shan (NO de China). [Rhizophyllum Lindström, 1866 (Rugosa) of the Lower Devonian (Lochkovian) from the SE of the Tian-Shan Mountains (NW China)]. Revista Española de Paleontología, 13 (2), 197-208. ISSN 0213-6937.

\begin{abstract}
In this study five species of the genus Rhizophy/lum, from the Arpishmebulaq Formation (Lochkovian) in the SE of the Tian-Shan Mountains (Xinjiang province, NW China), are described. Of these species, Rhizophy/lum extensum is new; $R$. sp. A and $R$. sp. B are probably new as well, but the lack of more abundant and suitable material allows only, for the moment, their description in open nomenclature. $R$. hedini (Regnell) was only known by its external morphology, but now the internal structures are described for the first time. Finally, $R$. cf. enorme (Etheridge) has never been reported in China and its presence in the Arpishmebulaq area confirms biogeographical relationships during the Lochkovian with the Ural Montains, Salair, Central Asia (Altai) and Eastern Australia.
\end{abstract}

Keywords: Rugosa, Taxonomy, Biogeography, Lower Devonian (Lochkovian), Arpishmebulaq Formation, Xinjiang Province, China.

\section{RESUMEN}

En el presente trabajo se describen varias especies del género Rhizophyllum Lindström, procedentes de la Formación Arpishmebulaq (Lochkoviense), en el SE de las Montañas de Tian-Shan (provincia de Xinjiang, NO de China). Las descripciones realizadas comprenden cinco especies, de las cuales Rhizophyllum extensum se propone como nueva, $R$. sp. A y $R$. sp. B son también, probablemente, nuevas, pero la falta de material más abundante y mejor conservado hace que las mantengamos, por el momento, en nomenclatura abierta. $R$. hedini (Regnell) era conocida solo por su morfología externa y ahora se describen por primera vez sus estructuras internas. Finalmente, $R$. cf. enorme (Etheridge) no habia sido citada nunca en China y su presencia en el área de Arpishmebulaq confirma relaciones biogeográficas durante el Lochkoviense con los Montes Urales, Salair, Asia Central (Altai) y Este de Australia.

Palabras clave: Rugosos, Taxonomía, Biogeografía, Devónico Inferior (Lochkoviense), Formación Arpishmebulaq, Provincia de Xinjiang, China.

\section{INTRODUCCIÓN}

El género Rhizophyllum Lindström se caracteriza, al igual que Calceola Lamarck, por poseer una morfología peculiar, similar a la de una babucha. Las coralitas tienen un lado aplanado, son semicirculares o semielípticas en sección transversal y los cálices estan cubiertos por un opérculo.

Numerosas especies de Rhizophyllum han sido descritas o mencionadas fundamentalmente en el Silúrico de distintas partes del mundo (Römer, 1854, 1856; de Koninck, 1876-1877; Lyon, 1879; Lindström, 1883;
Shearsby, 1905, 1906; Reed, 1924; Grabau, 1925; Soshkina, 1937; Sugiyama, 1940; Wang, 1948; Sutherland, 1965; Ivanovskiy, 1965; entre otros), pero algunas de ellas han perdurado o se han encontrado por primera vez en el Devónico Inferior (Bayle, 1878; Lindström, 1883; Pocta, 1902; Etheridge, 1903; Soshkina, 1937, 1949, 1952; Termier y Termier, 1950; Bulvanker, 1958; Oliver, 1964; entre otros) y, posiblemente, en la parte baja del Devónico Medio (Spasskiy, 1960; Hill y Jell, 1969; Hill, 1981).

Inicialmente, este género fue considerado por muchos geólogos chinos como un coral típicamente silúrico. Sin 


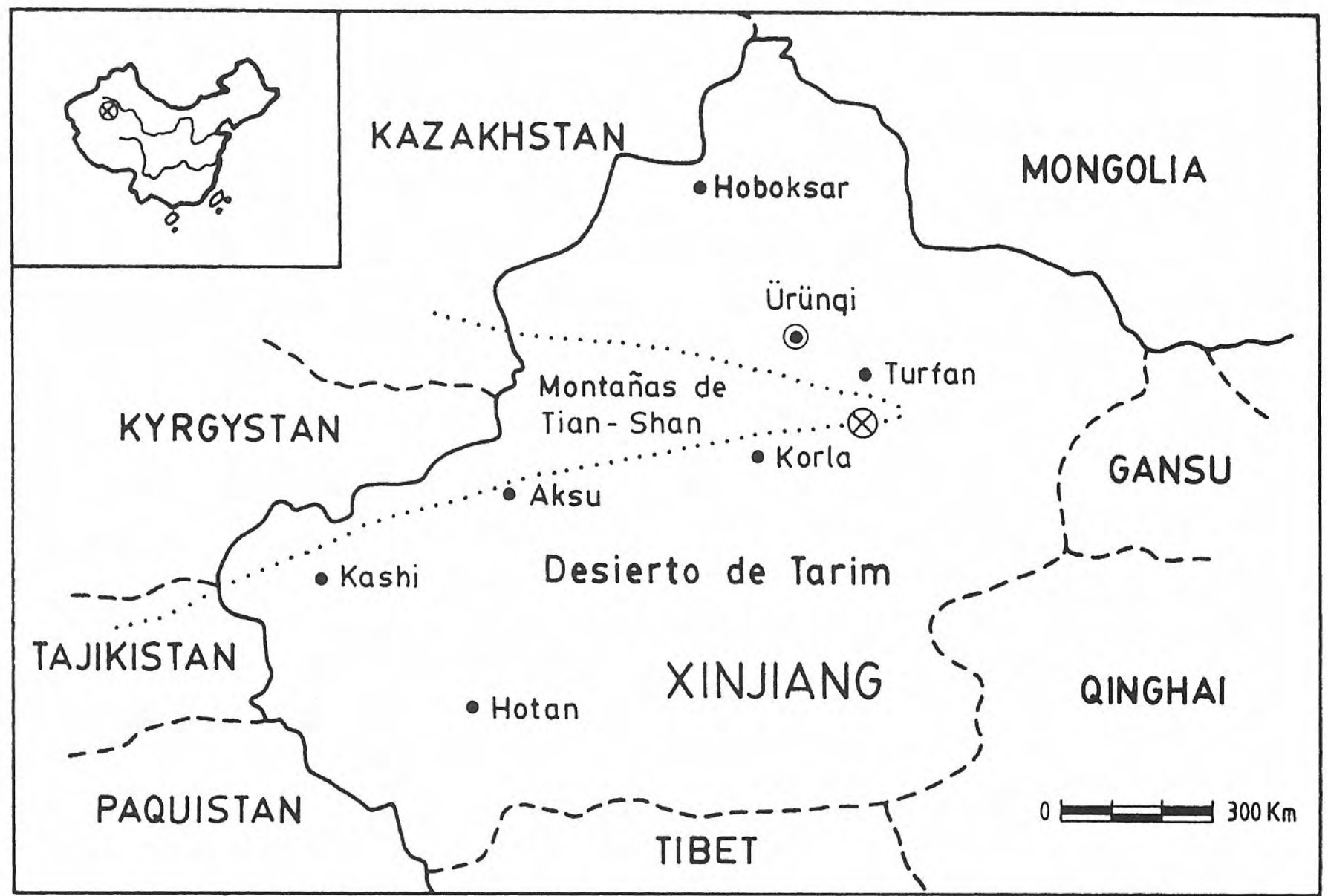

Figura 1. Localización geográfica de la sección de Arpishmebulaq (provincia de Xinjiang, NO de China).

embargo, los hallazgos realizados a lo largo del tiempo, no solo en China sino también en muchas otras partes del mundo, han permitido establecer una distribución estratigráfica del taxón de mucho mayor alcance, desde el Silúrico (Llandoveriense Superior) hasta el Devónico Inferior (Emsiense) y, posiblemente, el Devónico Medio (Eifeliense Inferior).

Los descubrimientos realizados hasta ahora en China, relacionados con especies del género Rhizophyllum, no son muy numerosos y se reparten entre el Llandoveriense y el Emsiense Inferior. La mayor parte de especies descritas corresponde a nuevos taxones y su registro, hasta el momento, es el siguiente:

- Rhizophyllum sinense Lindström, 1883; Llandoveriense; Guangyuan (prov. de Sichuan)

- R. minor Grabau, 1925; Llandoveriense; Lojoping (prov. de Hubei)

- $R$. changi Wang, 1948; LlandoverienseWenlockiense; Qiaojia (prov. de Yunnan)

- $R$. ningqiangense Ge y Yü, 1974; Llandoveriense; Ningqiang (prov. de Shaanxi)

- R. gotlandicum Römer, 1856. Cao, 1975; Llandoveriense; Ningqiang (prov. de Shaanxi)

- R. yanbianense He, 1978; LlandoverienseWenlockiense; Yanbian (prov. de Sichuan)

- $R$. zhifangense Cai, 1981; Ludloviense-Pridoliense; Barkol (prov. de Xinjiang)
- $R$. sp. Cao, in Cao y Ouyang, 1987; Pridoliense; Diebu (prov. de Gansu)

- R. junggarense Li y Liao, 1994; Wenlockiense; Hoboksar (prov. de Xinjiang)

- R. hedini (Regnell, 1941); Lochkoviense; Arpishmebulaq (prov. de Xinjiang)

- R. gotlandicum Römer, 1856. Guo, 1980; Lochkoviense; Erdougou (prov. de Jilin)

- R. ohanqi Guo, 1980; Pridoliense-Lochkoviense; Ohanqi (prov. de Mongolia Interior)

- R. sp. Guo, 1980; Pridoliense-Lochkoviense; Qiankenton (prov. de Mongolia Interior)

- $R$. applanatum Cai, in Cai y Zeng, 1983; Devónico Inferior; Fuyun (prov. de Xinjiang)

- $R$. xinjiangense Cai, in Cai y Zeng, 1983; Devónico Inferior; Shanshan (prov. de Xinjiang)

- R. devonicum Yoh, 1988; Emsiense Inferior; Nanning (prov. de Guangxi)

En 1988 y 1992, uno de los autores de este trabajo (Liao, Weihua), en colaboración con otros colegas del Instituto de Geología y Paleontología de Nanjing (Academia Sinica), realizó trabajos de campo en la sección clásica de Arpishmebulaq (Formación Arpishmebulaq) y recolectó un conjunto de ejemplares pertenecientes al género Rhizophyllum, los cuales constituyen el material en que se basan las descripciones realizadas en la presente nota. Han sido identificadas 


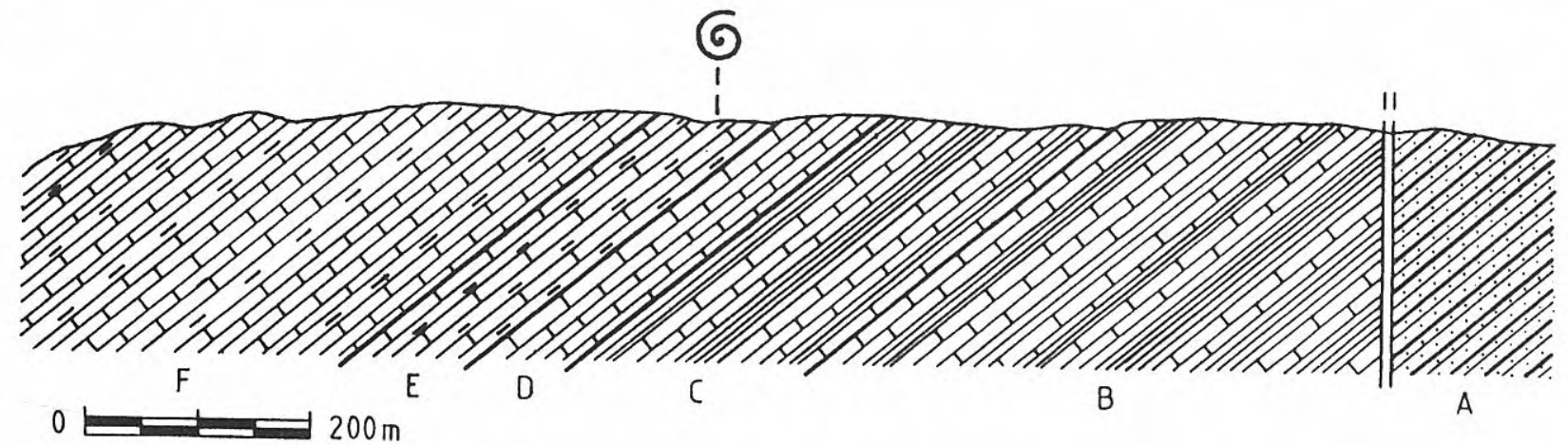

Figura 2. Unidades litoestratigráficas (A-F) distinguidas en la Formación Arpishmebulaq y localización de los yacimientos de Rhizophyllum (Unidad E).

cinco especies, Rhizophyllum hedini (Regnell), $R$. extensum n. sp., $R$. cf. enorme Etheridge, $R$. sp. A y $R$. sp. $\mathrm{B}$, de las cuales $R$. hedini es conocida únicamente en el Devónico Inferior (Lochkoviense) del SE de las Montañas de Tian-Shan y Tajikistan, y R. enorme ha sido citada en el Devónico Inferior (Lochkoviense-Emsiense) de los Urales, Salair, Asia Central y E de Australia. Estas distribuciones permiten confirmar, obviamente, unas relaciones biogeográficas estrechas durante el Devónico Inferior (Lochkoviense) entre el SE de Tian-Shan y los Montes Urales, Salair, Asia Central (Altai) y E de Australia.

\section{LOCALIZACIÓN GEOGRÁFICA Y ESTRATIGRÁFICA DE LA FAUNA ESTUDIADA}

La sección de Arpishmebulaq está situada en el borde SE de las Montañas de Tian-Shan (provincia de Xinjiang, NO de China), aproximadamente a unos $200 \mathrm{Km}$ al SE de Ürünqi, capital de la provincia (Fig. 1). En este punto geográfico, el Devónico Inferior (Lochkoviense) aparece bien expuesto, pero en contacto mecánico con rocas (esquistos y grauvacas), cuya edad precisa aún no ha sido establecida. La sección que corresponde al Devónico Inferior (Formación Arpishmebulaq) tiene un espesor de 410 m y está constituida, básicamente, por una alternancia de niveles calcáreos y lutíticos (Fig. 2), que han suministrado abundantes ejemplares de corales rugosos y tabulados, braquiópodos, algunos trilobites y, recientemente, también de conodontos.

Norin $(1937,1941)$ dividió originalmente la Formación Arpishmebulaq en seis Unidades (A a F) (Fig. 2), que denominó Divisiones, y sugirió, basándose en datos de Regnell (1941), que las Unidades A a E pertenecían al Silúrico Superior, mientras que la Unidad F debía de corresponder al Devónico Inferior.
Los especímenes de Rhizophyllum descritos en el presente trabajo proceden de la Unidad E (División E de Norin op. cit.), la cual tiene un espesor de $50 \mathrm{~m}$ y está constituida por lutitas arcillosas y calcáreas (Fig. 2), fácilmente distinguibles sobre el terreno por su color achocolatado. Recientemente, Liao y Xia (1996) han encontrado faunas de conodontos, concentradas en diferentes niveles de las Unidades D y F, con el hallazgo de especies o subespecies tales como Ozarkodina remscheidensis remscheidensis (Ziegler), Pandorinellina optima (Moskalenko) y Amydrotaxis praejohnsoni Murphy y Sringer, que permiten deducir una edad Lochkoviense Superior para los niveles con Rhizophyllum.

\section{DESCRIPCIONES SISTEMÁTICAS}

La clasificación seguida para este peculiar grupo de corales rugosos es la establecida recientemente por Weyer (1996).

Todos los especímenes proceden de la Formación Arpishmebulaq (Unidad E), extremo SE de las Montañas de Tian-Shan (provincia de Xinjiang, NO de China).

Los ejemplares y láminas delgadas están depositados en el Instituto de Geología y Paleontología de Nanjing (Academia Sinica), República Popular de China.

\section{ORDEN RUGOSA Edwards y Haime, 1850 \\ Familia Calceolidae King, 1846 \\ Subfamilia Calceolinae King, 1846 \\ Género Rhizophyllum Lindström, 1866}

Especie-tipo: Calceola gotlandica Römer, 1856.

\section{Diagnosis}

Coral rugoso, por lo general, solitario. Coralita con forma de babucha y cáliz de contorno subsemicircular, 


\begin{tabular}{|c|c|c|c|c|c|c|}
\hline $\begin{array}{c}\text { medidas } \\
\text { (mm) }\end{array}$ & 129089 & 129094 & 129095 & 129096 & 129097 & 129098 \\
\hline DM & 23 & 28 & 23 & 25 & 22 & 21 \\
\hline Dm & 17 & 10 & 16 & 16 & 10 & 15 \\
\hline Dm/DM & & 0.35 & 0,5 & 0,64 & 0,45 & 0,71 \\
\hline L & & $40(+8)$ & $25(+5)$ & $32(+6)$ & $26(+10)$ & 40 \\
\hline L/DM & & 1,7 & 1,3 & 1,45 & 1,6 & 1,9 \\
\hline L1 & 2,5 & 2 & & & & 2 \\
\hline SA & 0,8 & 1 & & & & 1 \\
\hline Sc & 0,8 & & & & & \\
\hline LA & 4 & 2,2 & & & & 3 \\
\hline DT & 12 & 12 & & 12 & 10 & \\
\hline Dt & 6 & 3 & & 5 & 3 & \\
\hline LA/Dm & & 0,2 & & & & 0,2 \\
\hline LA/L1 & & 1,1 & & & & 1,5 \\
\hline
\end{tabular}

Tabla 1. Dimensiones más representativas de los especímenes de Rhizophyllum hedini (Regnell). El significado de los símbolos utilizados es el siguiente: DM - diámetro mayor; Dm - diámetro menor; L - longitud; L1 - longitud máxima de los metaseptos; LA - longitud del septo antípoda; SA - espesor de la estereozona en el sector antípoda; Sc - espesor de la estereozona en el sector cardinal; DT - anchura mayor del tabulario; Dt anchura menor del tabulario.

con opérculo de denso esclerénquima. Prolongaciones radicales tubulares dispuestas irregularmente sobre el lado aplanado (antípoda) de la coralita. Septos, desarrollados a lo largo del lado aplanado de la coralita, incompletos y constituidos por trabéculas aisladas, cortas y finas. En ocasiones se disponen a modo de espinas radiales sobre disepimentos y tabelas. A veces, los septos se espesan y coalescen radial y lateralmente para formar una estereozona periférica más o menos densa sobre el lado antípoda o sobre ambos lados (cardinal y antípoda) de la coralita. Disepimentos y tabelas numerosos, difíciles de distinguir unos de otros.

\section{Observaciones}

Lindström (1883) consideró Platyphyllum sinense Lindström genéricamente distinguible de Rhizophyllum por el mayor desarrollo de sus septos, los cuales alcanzan la región axial. Hill y Jell (1969), tras analizar cuidadosamente dos sintipos de Platyphyllum sinense procedentes de la colección de Lindström, opinan, sin embargo, que las diferencias morfológicas entre Platyphyllum y Rhizophyllum no son suficientes para mantener la autonomía de los dos géneros y consideran Platyphyllum como un sinónimo subjetivo de Rhizophyllum.

Rhizophyllum y Calceola poseen coralitas cuya morfología externa es prácticamente similar; sin embargo, existen marcadas diferencias entre ambos taxones por lo que respecta a las estructuras que rellenan la cavidad interna de la coralita. Rhizophyllum posee su lumen totalmente colmado por tabelas y disepimentos vesiculares, mientras que Calceola está desprovisto de estas estructuras y su lumen está relleno completamente o casi por esclerénquima, dispuesto en láminas paralelas a la base del cáliz, en el que estan incluidas trabéculas de segmentos septales. Además, las coralitas de Calceola no poseen prolongaciones radicales.

\section{Distribución}

Silúrico Inferior (Llandoveriense)-Devónico Inferior (Emsiense); prácticamente cosmopolita (Europa, Asia, Norteamérica, Este de Australia). Posiblemente Devónico Medio (Eifeliense Inferior); Asia Central (Altai) y E de Australia.

\section{Rhizophyllum hedini (Regnell, 1941)}

Lám. I, figs. 1-9; Lám. II, figs. 1-4

\footnotetext{
* 1941 Teratophyllum hedini Regnell, Lám. 1, figs. 10 a-b.

. 1971 Rhizophyllum enorme Lavrusevich, p. 38, Lám. 2, fig. 4; Lám. 4, fig. 4.
}

Material: Seis ejemplares en diferentes estados de conservación. Especímenes 129089, del que se ha realizado una lámina delgada correspondiente a una sección transversal, 129094, del que se han efectuado dos láminas delgadas correspondientes a una sección transversal y una longitudinal (Lám. II, figs. 1-2), 129095 (Lám. I, figs. 1-3), 129096 (Lám. I, figs. 4-6), 129097 (Lám. I, figs. 7-9) y 129098, del que también se han hecho dos láminas delgadas correspondientes a una sección transversal y una longitudinal (Lám. II, figs. 3-4); SE de las Montañas de Tian-Shan, Formación Arpishmebulaq (Unidad E).

\section{Diagnosis (enmendada aquí)}

Rhizophyllum solitario, de tamaño medio, alcanzando una longitud de 30-48 mm, un diámetro mayor de 21-28

\section{Lámina I}

Todos los ejemplares proceden de la Formación Arpishmebulaq (Unidad E), situada en el área de Arpishmebulaq (SE de Ürünqi, capital de la provincia de Xinjiang), en el borde SE de las Montañas de Tian-Shan (NO de China). Todas las figuras x 1.

1-9 Rhizophyllum hedini (Regnell). 1-3, espécimen 129095, vistas cardinal, antípoda y lateral. 4-6, espécimen 129096, vistas cardinal, antípoda y lateral. 7-9, espécimen 129097, vistas cardinal, antípoda y lateral.

10-12 Rhizophyllum cf. enorme Etheridge. Espécimen 129090, vistas cardinal, antípoda y lateral.
13-18 Rhizophyllum extensum n. sp. 13-15, Paratipo 129102, vistas cardinal, antípoda y lateral. 16-18, Holotipo 129101, vistas cardinal, antípoda y lateral.

19-20 Rhizophyllum sp. A. Espécimen 129103, vistas cardinal, antípoda y lateral.

21-23 Rhizophyllum sp. B. Espécimen 129091, vistas cardinal, antípoda y lateral. 
Lámina I

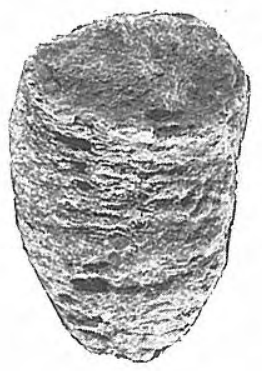

1

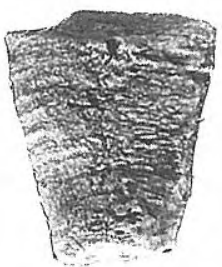

7

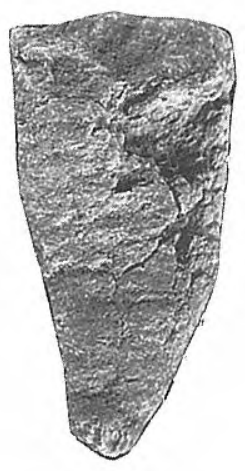

13

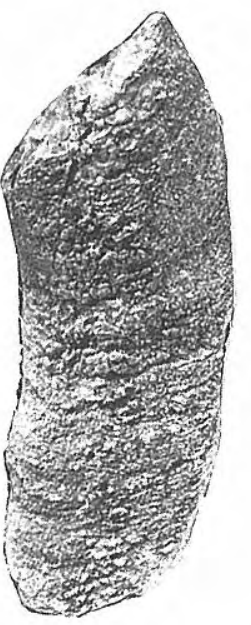

16

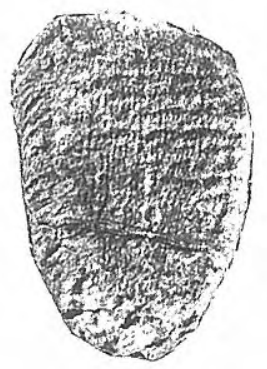

2

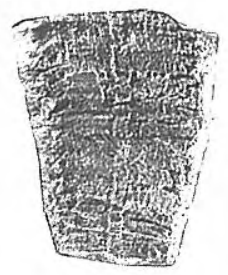

8

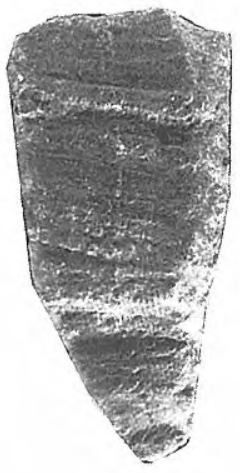

14

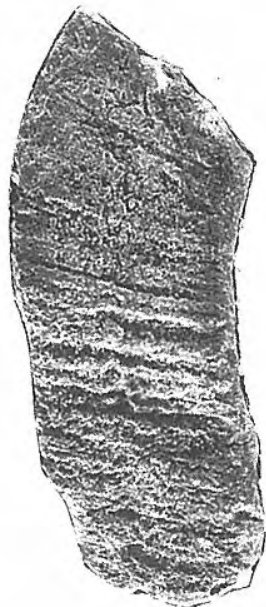

17

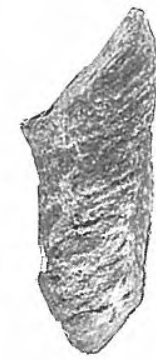

3

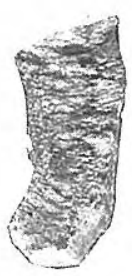

9

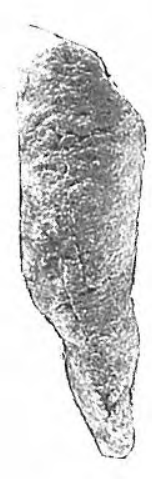

15

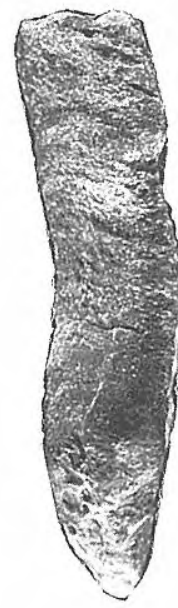

18

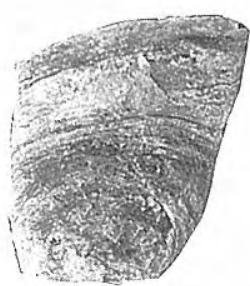

4

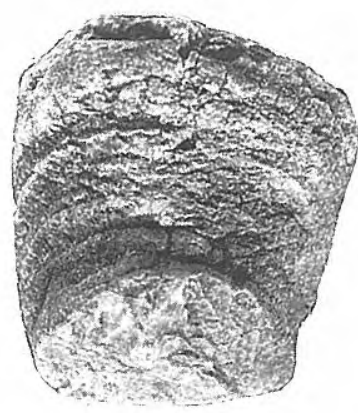

10

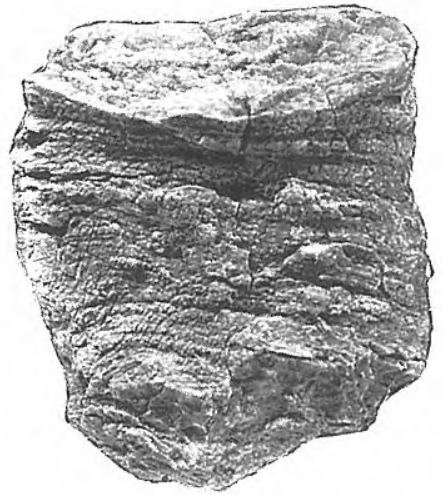

19

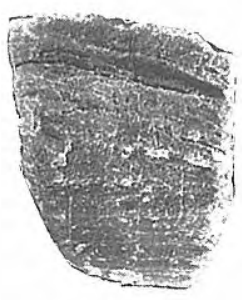

5

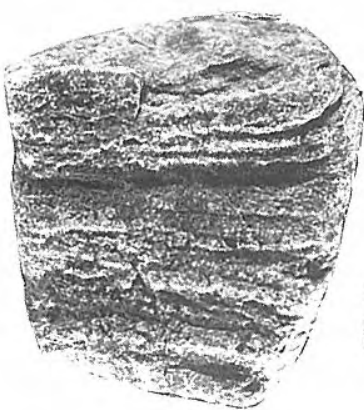

11

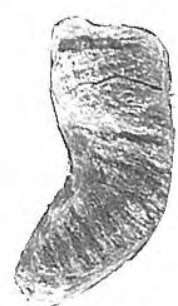

6

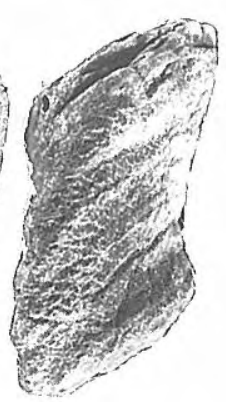

12

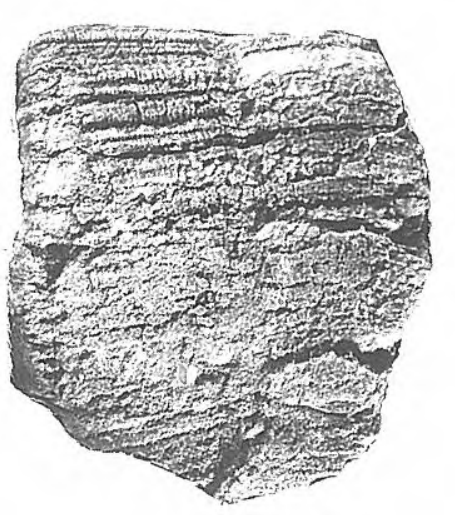

20

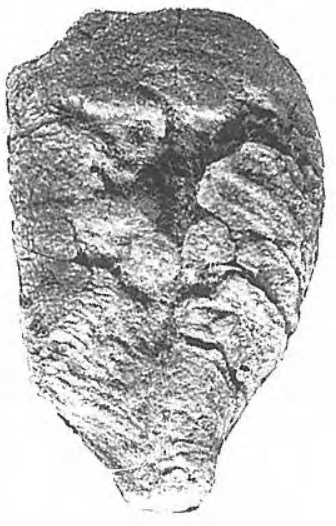

21

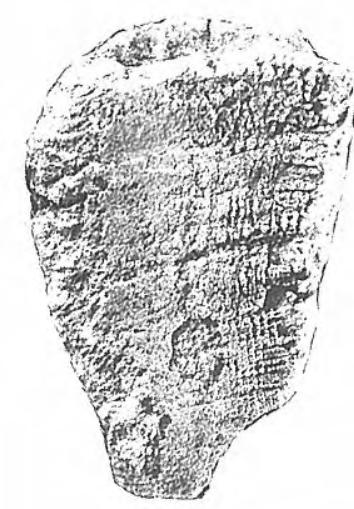

22

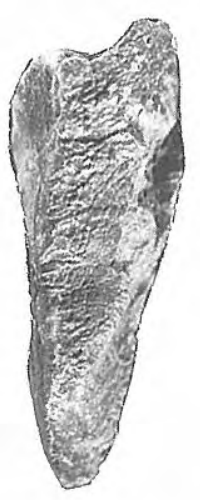

23

Revista Española de Paleontología, 13 (2), 1998. 
mm y un ángulo apical inicial oscilando entre $40^{\circ}-50^{\circ}$. Septo antípoda de mayor espesor y longitud que los metaseptos que lo flanquean. Estereozona antípoda espesa, de 0,8 a 1,4 mm. Tabulario estrecho, formado por tabelas con morfología variable, difíciles de distinguir de disepimentos.

\section{Descripción}

Coral solitario, de tamaño medio, generalmente recto, aunque algunos especímenes muestran la parte apical de la coralita curvada hacia la región cardinal. En sección transversal, se puede observar que la superficie antípoda es aplanada y la cardinal más o menos convexa (Lám. II, figs. 1,3). Los ángulos laterales entre ambas superficies son redondeados y el contorno que proporciona la sección transversal es semielíptico. En la mayor parte de las coralitas, la epiteca está desgastada y únicamente en la superficie aplanada de las mismas, correspondiente a la región antípoda, se muestran finas arrugas de crecimiento y estrías longitudinales redondeadas (Lám. I, figs. 2, 8). Cuando la epiteca falta en su totalidad, en la superficie cardinal se observa gran número de disepimentos vesiculares. Los cálices parecen ser poco profundos. La superficie aplanada (antípoda) de algunas coralitas presenta pequeñas prolongaciones, probablemente correspondientes a procesos radicales (Lám. I, figs. 5, 8). El ejemplar más grande disponible tiene un diámetro mayor de $28 \mathrm{~mm}$, una longitud de aproximadamente $48 \mathrm{~mm}$ y un ángulo apical inicial de $40^{\circ}$. La variación del tamaño y de otras características (Strusz, 1970, p. 282-283) tenidas en cuenta para la definición de las especies del género Rhizophyllum se muestran en la Tabla 1.

El septo antípoda se diferencia de los septos que lo flanquean por su mayor espesor y longitud. El resto de septos del sector antípoda decrece gradualmente en longitud desde el septo antípoda hasta los extremos del cáliz. En aquellos lugares del sector antípoda donde los septos se conservan bien, se cuentan 2-3 septos cada $5 \mathrm{~mm}$. En el sector cardinal los septos estan representados por un número variable de espinas trabeculares finas y cortas sobre las superficies disepimentales (Lám. II, figs. 6, 8).

El disepimentario es más amplio en la región antípoda que en la cardinal. Los disepimentos son pequeños, globosos y alargados. Los que están situados en el lado antípoda son horizontales en las proximidades de la muralla interna, mientras que hacia el interior de la coralita se van inclinando progresivamente hasta casi verticalizarse en los puntos de contacto con el tabulario (Lám. II, figs. 2, 4). En el lado cardinal, los disepimentos son alargados y están fuertemente inclinados en dirección aboral. El tabulario es muy estrecho y está constituido por tabelas con morfologías bastante variables. Algunas son completas y planas, otras son incompletas y vesiculosas; más raramente cóncavas (Lám. II, fig. 4).

El espesor de la estereozona en el sector antípoda oscila entre 0,8 y $1,4 \mathrm{~mm}$ y desde ella los septos se proyectan hacia el interior de la coralita, no más allá de 0,5-1,6 mm.

\section{Discusión}

Los especímenes descritos han sido recolectados en los mismos niveles (Unidad E de la Formación Arpishmebulaq) que el holotipo de Rhizophyllum hedini (Regnell) y muestran una similitud total, en cuanto a tamaño y morfología, con el único ejemplar descrito y figurado por Regnell (1941, Lám. 1, fig. 10). Dado que Regnell no describió las características internas de $R$. hedini, ésta es la primera vez que se da a conocer la estructura interna del taxón, lo cual permite una enmienda de su diagnosis.

$R$. hedini se diferencia de $R$. enorme Etheridge en su menor tamaño y ángulo apical, en la no conservación de la depresión axial ("axial pit") y en la diferente disposición y morfología de tabelas y tabulario.

La especie descrita e identificada como $R$. enorme por Lavrusevich (1971, p. 38, Lám. 2, fig. 4, Lám. 4, fig. 4), procedente de Tajikistan, pertenece, en nuestra opinión, a $R$. hedini, ya que tanto el tamaño de la coralita, como la disposición de estructuras internas coinciden sensiblemente.

\section{Distribución geográfica y estratigráfica}

Sección de Arpishmebulaq, SE de las Montañas de TianShan (prov. de Xinjiang, NO de China) y Tajikistan; Formación Arpishmebulaq (Unidad E), Devónico Inferior (Lochkoviense Superior).

\section{Rhizophyllum extensum $\mathrm{n}$. sp.} Lám. I, figs. 13-18; Lám. II, figs. 5-9

Holotipo: Espécimen 129101 (Lám. I, figs. 16-18), del que se han realizado dos secciones transversales y una sección longitudinal (Lám. II, figs. 5-7).

Estrato y localidad típicos: Formación Arpishmebulaq (Unidad E), Devónico Inferior (Lochkoviense Superior); Sección de Arpishmebulaq, SE de las Montañas de TianShan (provincia de Xinjiang, NO de China).

Material: Tres ejemplares adicionales (Paratipos). Especímenes 129099, del que se han efectuado dos láminas delgadas, correspondientes a una sección transversal y una longitudinal (Lám. II, figs. 8-9), 129100 y 129102 (Lám. I, figs. 13-15), de la misma localidad y nivel estratigráfico.

Derivatio nominis: Proviene del latin extentus-a-um (o tensum) y hace referencia a la forma alargada de las coralitas.

\section{Diagnosis}

Especie de Rhizophyllum que alcanza una longitud de 50-60 mm, un diámetro mayor de $25 \mathrm{~mm}$ y un ángulo apical inicial que oscila entre $25^{\circ}-37^{\circ}$. Estereozona delgada, solo visible en el sector antípoda, de la que se proyectan septos muy cortos. En el sector cardinal se observan espinas septales finas sobre las superficies disepimentales. Tabulario muy estrecho, constituido por tabelas cóncavas en forma de embudo.

Species of Rhizophyllum, 50-60 mm long, with a maximum major diameter of $25 \mathrm{~mm}$ and an initial apical 


\begin{tabular}{|c|c|c|c|c|}
\hline medidas(mm) & 129099 & 129100 & 129101 & 129102 \\
\hline $\mathrm{DM}$ & 26 & 20 & 25 & 24 \\
\hline $\mathrm{Dm}$ & 10 & 10 & 9 & 15 \\
\hline $\mathrm{Dm} / \mathrm{DM}$ & 0,38 & 0,5 & 0,36 & 0,62 \\
\hline $\mathrm{L}$ & 50 & 40 & $55(+10)$ & 49 \\
\hline $\mathrm{L} / \mathrm{DM}$ & 1,9 & 2 & 2,6 & 2 \\
\hline $\mathrm{L} 1$ & 1 & & 1 & \\
\hline $\mathrm{SA}$ & 0,9 & & 0,5 & \\
\hline $\mathrm{LA}$ & 2 & & 1,5 & \\
\hline $\mathrm{DT}$ & & & & 9 \\
\hline $\mathrm{Dt}$ & & & & 3 \\
\hline $\mathrm{LA} / \mathrm{Dm}$ & 0,2 & & 0,16 & \\
\hline $\mathrm{LA} / \mathrm{L} 1$ & 2 & & 1,5 & \\
\hline
\end{tabular}

Tabla 2. Dimensiones más representativas de los especímenes de Rhizophyllum extensum n. sp. Símbolos como en la Tabla 1.

angle about $25^{\circ}-37^{\circ}$. Stereozone thin, only visible in the counter side, and septa along the counter wall usually very short. In the cardinal side, septal spines on the dissepiments short and fine. Tabularium very narrow, constituted by funnel-shaped tabellae.

\section{Descripción}

La superficie cardinal de las coralitas está desgastada y en algunos casos muestra numerosos disepimentos vesiculares (Lám. I, fig. 16); sin embargo, la epiteca de la superficie antípoda está recorrida longitudinalmente por estrías finas y redondeadas, separadas por surcos estrechos. Las estrías tienen un espesor uniforme y, perpendicularmente, están cruzadas por arrugas de crecimiento finas y gruesas alternando de manera irregular (Lám. I, figs. 14-17). Los cálices parecen poco profundos. El ejemplar de mayor tamaño tiene un diámetro mayor de $25 \mathrm{~mm}$, una longitud de aproximadamente $65 \mathrm{~mm}$ y un ángulo apical de $25^{\circ}$. La variación en tamaño y otras características propias de los ejemplares de esta especie se muestran en la Tabla 2.

El septo antípoda parece poseer un tamaño ligeramente superior al de los metaseptos vecinos, pero la longitud del resto de los metaseptos decrece de forma gradual hacia los extremos del cáliz (Lám. II, figs. 6, 8). En el sector antípoda, donde los septos están bien conservados, se cuentan 3 septos cada $5 \mathrm{~mm}$, sin embargo, en el sector cardinal, los septos están representados por finas y cortas espinas trabeculares, situadas sobre la superficie de disepimentos próximos a la muralla (Lám. II, figs. 6, 8). El disepimentario es algo más ancho en la región cardinal que en la región antípoda y está constituido por disepimentos pequeños, globosos y alargados. Los que se disponen sobre el lado antípoda son horizontales hacia el exterior de la coralita y se inclinan progresivamente en dirección aboral hacia el tabulario; los del lado cardinal son alargados y muy inclinados hacia la región axial de la coralita (Lám. II, figs. 7, 9). El tabulario es muy estrecho y está formado por tabelas cóncavas, con forma de embudo (Lám. II, figs. 7, 9).

El espesor de la estereozona en el sector antípoda es 0,5$0,9 \mathrm{~mm}$ y los septos se proyectan ligeramente desde ella hacia el interior del disepimentario.

\section{Discusión}

La nueva especie se parece a Rhizophyllum hedini (Regnell) por lo que respecta a las medidas del diámetro mayor de la coralita, anchura del tabulario y número de septos por cada $5 \mathrm{~mm}$; sin embargo, difiere ampliamente de la especie de Regnell por la longitud de la coralita muy superior, ángulo apical, por lo general, más agudo, estereozona antípoda menos espesa y tabelas mucho más embudadas.

\section{Distribución geográfica y estratigráfica}

Sección de Arpishmebulaq, SE de las Montañas de TianShan (prov. de Xinjiang, NO de China); Formación Arpishmebulaq (Unidad E), Devónico Inferior (Lochkoviense Superior).

\section{Rhizophyllum cf. enorme Etheridge, 1903 Lám. I, figs. 10-12; Lám. II, figs. 10-13}

\section{*1903 Rhizophyllum enorme Etheridge, p. 232, Lám. 47. \\ 1958 Rhizophyllum enorme Etheridge; Bulvanker, p. 46, Lám. 17. \\ 1968 Rhizophyllum enorme Etheridge; Besprozvannykh, p. 143, Lám. 64, fig. 4.}

Material: Tres ejemplares, de los que dos corresponden a fragmentos de coralitas. Especímenes 129087 (Lám. II, fig. 10), 129088 (Lám. II, fig. 11), ambos correspondientes a secciones transversales, y 129090 (Lám. I, figs. 10-12), del que se han realizado una sección transversal y una longitudinal (Lám. II, figs. 12-13); SE de las Montañas de Tian-Shan, Formación Arpishmebulaq (Unidad E).

\section{Descripción}

Coral solitario, aparentemente recto, aunque uno de los especímenes (Lám. I, fig. 10) muestra la región apical curvada en dirección cardinal. En sección transversal, la superficie antípoda es aplanada, la cardinal convexa y los ángulos entre ambas superficies más o menos redondeados, proporcionando, en conjunto, un contorno semicircular o semielíptico. Tanto la superficie aplanada (antípoda), como la convexa (cardinal) de la coralita, están atravesadas por arrugas de crecimiento finas y gruesas alternando irregularmente (Lám. I, fig. 11). El ejemplar de mayor tamaño tiene un diámetro mayor de $55 \mathrm{~mm}$, pero, por tratarse de un fragmento, no se conoce su longitud ni el ángulo apical. La variación de las medidas que han podido ser tomadas en los ejemplares a disposición se muestra en la Tabla 3.

Los septos que aparecen en el sector antípoda están, usualmente, bastante espaciados y se proyectan desde la estereozona hacia el interior del disepimentario. El septo antípoda es algo más grueso y ligeramente más largo que los metaseptos vecinos, pero la longitud de los demás metaseptos va decreciendo progresivamente hacia los extremos del cáliz. Se cuentan 5-8 septos por cada $5 \mathrm{~mm}$. En el sector cardinal, los septos se disponen sobre las superficies disepimentales a modo de cortas y finas espinas (Lám. II, fig. 12). 


\begin{tabular}{|c|c|c|c|}
\hline medidas $(\mathrm{mm})$ & 129087 & 129088 & 129090 \\
\hline $\mathrm{DM}$ & 55 & 31 & 35 \\
\hline $\mathrm{Dm}$ & 24 & 12 & 28 \\
\hline $\mathrm{Dm} / \mathrm{DM}$ & 0,43 & 0,38 & 0,8 \\
\hline $\mathrm{L}$ & & & $34(+16)$ \\
\hline $\mathrm{L} / \mathrm{DM}$ & & & 1,43 \\
\hline $\mathrm{L} 1$ & 2,5 & 2 & 1 \\
\hline $\mathrm{SA}$ & 1 & 1,2 & 0,5 \\
\hline $\mathrm{Sc}$ & 0,1 & 0,3 & \\
\hline $\mathrm{LA}$ & 3 & 2,5 & 1 \\
\hline $\mathrm{DT}$ & 26 & 17 & 17 \\
\hline $\mathrm{Dt}$ & 12 & 7 & 7 \\
\hline $\mathrm{LA} / \mathrm{Dm}$ & 0,12 & 0,2 & 0,03 \\
\hline
\end{tabular}

Tabla 3. Dimensiones más representativas de los especímenes de Rhizophyllum cf. enorme Etheridge. Símbolos como en la Tabla 1.

El disepimentario de la región cardinal es mucho más amplio que el de la región antípoda y está formado por disepimentos de pequeño tamaño, alargados o globosos. Los correspondientes al sector antípoda son globosos y horizontales hacia el exterior de la coralita y se inclinan ligeramente cuando contactan con el tabulario, mientras que los del sector cardinal son alargados e inclinados en dirección aboral. El tabulario es muy estrecho y está constituido por tabelas globosas, horizontales o ligeramente cóncavas (Lám. II, fig. 13).

El espesor de la estereozona en el sector antípoda oscila entre 1-1,2 mm, mientras que en el sector cardinal es mucho más fina y no supera los $0,3 \mathrm{~mm}$.

\section{Discusión}

Nuestros especímenes descritos muestran bastantes semejanzas con los tipos australianos de Rhizophyllum enorme por lo que respecta a las medidas del diámetro mayor, longitud y ángulo apical de las coralitas. Sin embargo, en sección longitudinal, la disposición y tamaño de los disepimentos, tanto en el sector antípoda como en el cardinal, la débil concavidad de las tabelas en el estrecho tabulario y la falta de dilataciones septales sobre la superficie de las vesículas, diferencian nuestros ejemplares de los tipos de $R$. enorme. La escasez de material hace que dispongamos tan solo de una sección longitudinal que, obviamente, no nos permite analizar la variabilidad de las estructuras internas. Por esta razón, a falta de más ejemplares que nos proporcionen las evidencias precisas para una asignación segura, preferimos mantener el taxón en nomenclatura abierta.

Los especímenes rusos figurados como $R$. enorme por Bulvanker (1958, Lám. 17) y Besprozvannykh (1968, Lám. 64, fig. 4), son totalmente similares a los descritos en este trabajo en cuanto a tamaño, forma y estructuras internas.

\section{Distribución geográfica y estratitagráfica}

Sección de Arpishmebulaq, SE de las Montañas de TianShan (prov. de Xinjiang, NO de China); Formación Arpishmebulaq (Unidad E), Devónico Inferior (Lochkoviense Superior).

\section{Rhizophyllum sp. A}

Lám. I, figs. 19-20; Lám. II, figs. 14-15

Material: Dos ejemplares en mal estado de conservación. Especímenes 129103 (Lám. I, figs. 19-20) y 129104, del que se han realizado una sección transversal y una longitudinal (Lám. II, figs. 14-15); SE de las Montañas de Tian-Shan, Formación Arpishmebulaq (Unidad E).

\section{Descripción}

Coral solitario, aparentemente recto. En sección transversal, la superficie antípoda es aplanada, la cardinal suavemente convexa y los extremos del cáliz subangulosos. Contorno subsemielíptico. Sobre la superficie externa antípoda, no existen evidencias de prolongaciones radicales ni de otros medios de enraizamiento.

La superficie cardinal está bastante desgastada y muestra numerosos disepimentos vesiculares (Lám. I, fig. 19). La superficie antípoda, sin embargo, presenta una epiteca constituida por arrugas de crecimiento, dispuestas transversalmente a la coralita, y estrías longitudinales finas y redondeadas, separadas por surcos estrechos (Lám. I, fig. 20). Los cálices son poco profundos. El ejemplar de mayor tamaño tiene un diámetro de $40 \mathrm{~mm}$, una longitud de aproximadamente $55 \mathrm{~mm}$ y un ángulo apical que se sitúa en torno a los $60^{\circ}$. Las medidas más representativas de los ejemplares que se describen se muestran en la Tabla 4.

El septo antípoda tiene el mismo tamaño y espesor que los metaseptos vecinos, los cuales, conjuntamente con el resto de metaseptos del sector antípoda, disminuyen gradualmente de tamaño desde el septo antípoda hasta los

\section{Lámina II}

Todos los ejemplares proceden de la Formación Arpismebulaq (Unidad E), situada en el área de Arpishmebulaq (SE de Ürünqi, capital de la provincia de Xinjiang), en el borde SE de las Montañas de Tian-Shan (NO de China). Todas las figuras x 2.

1-4 Rhizophyllum hedini (Regnell). 1-2, espécimen 129094, secciones transversal y longitudinal. 3-4, espécimen 129098, secciones transversal y longitudinal.

5-9 Rhizophyllum extensum n. sp. 5-7, Holotipo 129101. 5, sección transversal, 6, sección transversal, 7, sección longitudinal. 8-9, Paratipo 129099, secciones transversal y longitudinal.
10-13 Rhizophyllum cf. enorme Etheridge. 10, espécimen 129087, sección transversal. 11, espécimen 129088, sección transversal. 12-13, espécimen 129090, secciones transversal y longitudinal.

14-15 Rhizophyllum sp. A. Espécimen 129104, secciones transversal y longitudinal.

16-17 Rhizophyllum sp. B. Espécimen 129091, secciones transversal y longitudinal. 
Lámina II
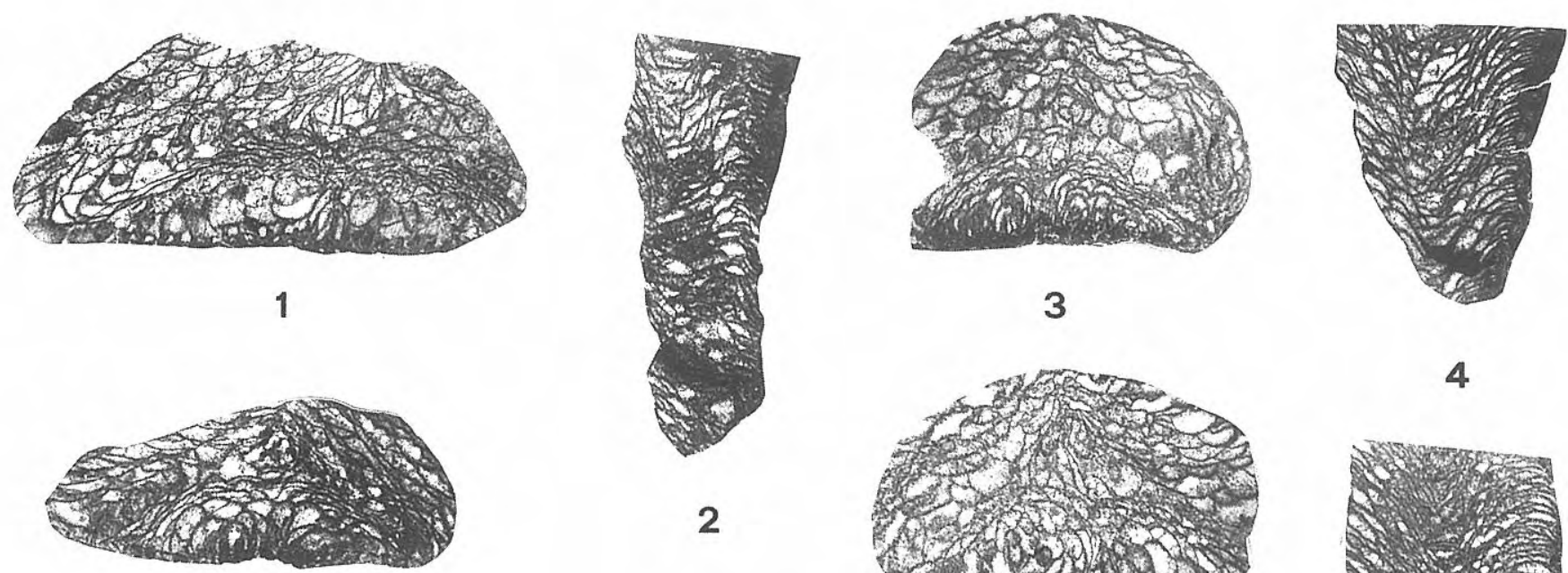

\section{3}

4

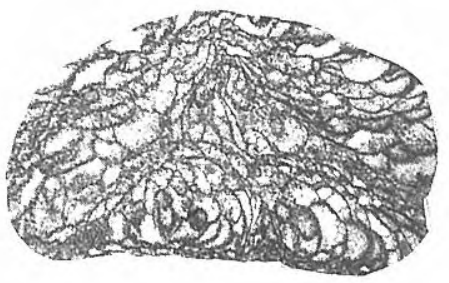

5
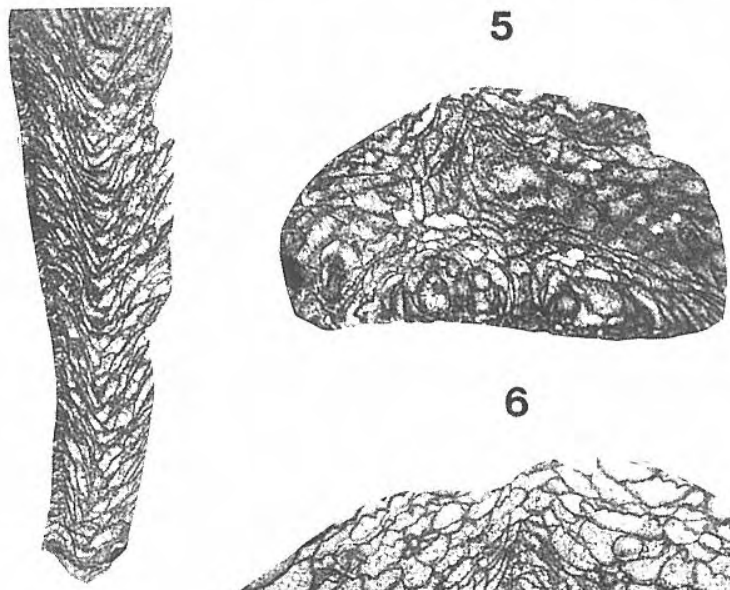

6
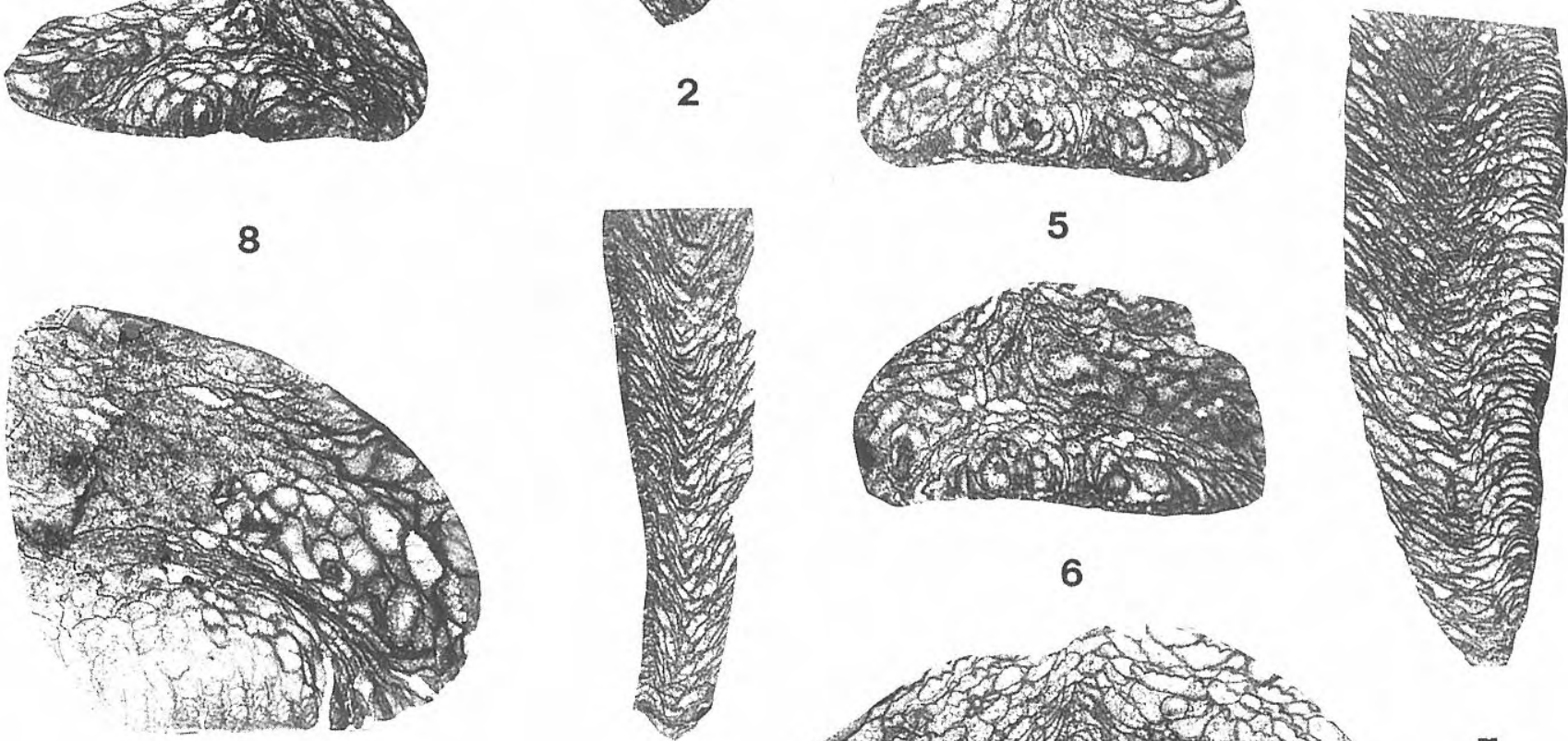

10

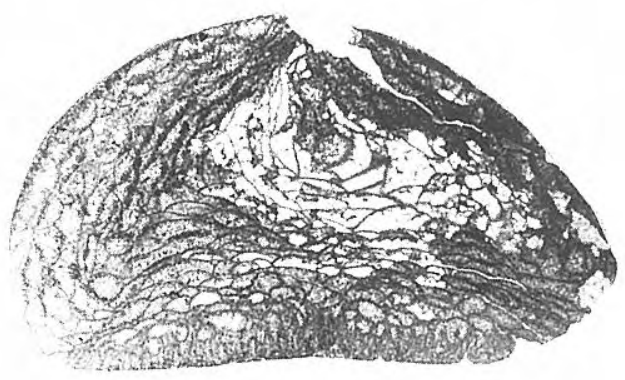

11

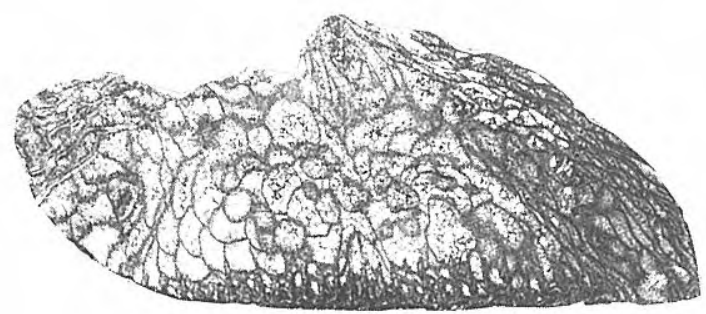

16

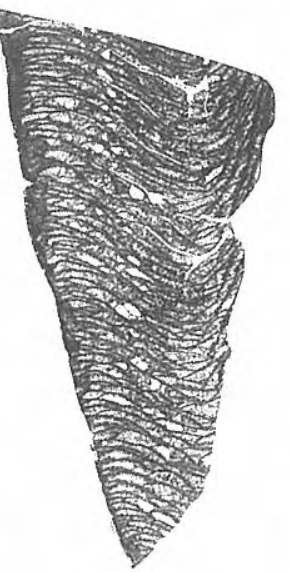

17
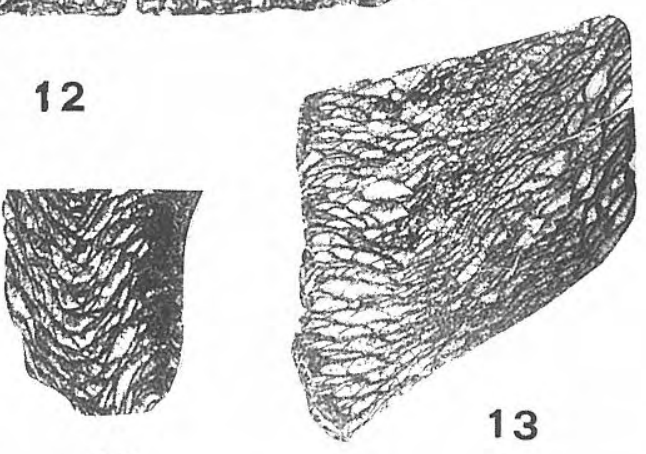

15

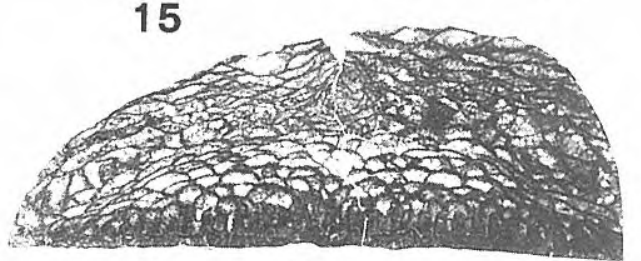

14

Revista Española de Paleontología, 13 (2), 1998. 
ángulos del cáliz (Lám. II, fig. 14). En este sector se cuentan 6 septos cada $5 \mathrm{~mm}$.

El disepimentario correspondiente a la sección antípoda es algo más amplio que el cardinal y está constituido por disepimentos de pequeño tamaño, globosos y alargados. En el sector antípoda son horizontales hacia el exterior de la coralita y casi se verticalizan en las proximidades del tabulario, mientras que en el sector cardinal son alargados y fuertemente inclinados en dirección aboral (Lám. II, fig. 15). El tabulario es relativamente amplio y está formado por tabelas cóncavas, en ocasiones con marcada forma de embudo.

La estereozona de la región antípoda está bien desarrollada y alcanza un espesor de $1 \mathrm{~mm}$.

\section{Discusión}

Los especímenes de este taxón muestran grandes semejanzas con los de $R$. cf. enorme, descrito anteriormente, en cuanto a las medidas de la coralita relacionadas con la longitud, diámetro mayor, ángulo apical, estereozona antípoda e, inclusó, è cuanto al número de septos cada $5 \mathrm{~mm}$. Sin embargo, existen diferencias en el contorno de la región cardinal del cáliz, subsemielíptico en $R$. sp. A, y en la disposición y morfología de los disepimentos y tabelas en sección longitudinal que, en nuestra opinión, son suficientes para distinguir esta especie de $R$. cf. enorme. El hecho de disponer de tan escaso material no nos permite por el momento realizar una asignación segura, por lo que estimamos conveniente mantener el taxón en nomenclatura abierta.

\section{Distribución geográfica y estratigráfica}

Sección de Arpishmebulaq, SE de las Montañas de TianShan (prov. de Xinjiang, NO de China); Formación Arpishmebulaq (Unidad E), Devónico Inferior (Lochkoviense Superior).

\section{Rhizophyllum sp. B}

\section{Lám. I, figs. 21-23; Lám.II, figs. 16-17}

Material: Un ejemplar con la región cardinal en deficiente estado de conservación. Espécimen 129091 (Lám. I, figs. 21-23), del que se han efectuado una sección transversal y una longitudinal (Lám. II, figs. 16-17); SE de las Montañas de Tian-Shan, Formación Arpishmebulaq (Unidad E).

\section{Descripción}

Coral solitario, aparentemente recto. En sección transversal, la superficie antípoda es aplanada, la cardinal convexa y los extremos del cáliz, donde convergen ambas superficies, son subangulosos. El contorno de la coralita en las proximidades del cáliz es subsemicircular. Aunque la superficie plana (antípoda) de la coralita está bastante desgastada, no parece que existan restos de prolongaciones relacionadas con procesos radicales.

Tanto la superficie cardinal como la antípoda muestran transversalmente finas arrugas de crecimiento, correspondientes a la epiteca, y, además, la superficie aplanada (antípoda) está recorrida por finas estrías longitudinales,

\begin{tabular}{|c|c|c|}
\hline medidas(mm) & 129103 & 129104 \\
\hline $\mathrm{DM}$ & 40 & 35 \\
\hline $\mathrm{Dm}$ & 13 & 16 \\
\hline $\mathrm{Dm} / \mathrm{DM}$ & 0,32 & 0,45 \\
\hline $\mathrm{L}$ & $40(+15)$ & $20(+15)$ \\
\hline $\mathrm{L} / \mathrm{DM}$ & 1,37 & 1 \\
\hline $\mathrm{L} 1$ & & 1,5 \\
\hline $\mathrm{SA}$ & & 1 \\
\hline $\mathrm{Sc}$ & & \\
\hline $\mathrm{LA}$ & & 1,5 \\
\hline $\mathrm{DT}$ & 15 & 15 \\
\hline $\mathrm{Dt}$ & 4,5 & 5 \\
\hline $\mathrm{LA} / \mathrm{Dm}$ & 0,1 & 0,1 \\
\hline $\mathrm{LA} / \mathrm{L} 1$ & & 0,1 \\
\hline
\end{tabular}

Tabla 4. Dimensiones más representativas de los dos especímenes de Rhizophyllum sp. A. Símbolos como en la Tabla 1.

separadas por surcos estrechos. El cáliz es poco profundo. El único ejemplar que poseemos tiene una longitud de $55 \mathrm{~mm}$, un diámetro mayor de $32 \mathrm{~mm}$ y un ángulo apical de aproximadamente $60^{\circ}$.

El septo antípoda es más largo, pero del mismo espesor que los metaseptos vecinos. Desde el septo antípoda hasta los extremos del cáliz, los metaseptos decrecen progresivamente en longitud y se pueden contar alrededor de 7 cada 5 $\mathrm{mm}$.

El disepimentario correspondiente al sector antípoda tiene una amplitud mucho mayor que el del sector cardinal. Está formado por disepimentos alargados y, en ocasiones, ligeramente globosos. En el sector antípoda son horizontales hacia el exterior de la coralita y se inclinan suavemente hacia el tabulario, mientras que en el sector cardinal están fuertemente inclinados en dirección aboral (Lám. II, fig. 17). El tabulario es bastante amplio y las tabelas son horizontales o levemente cóncavas. La estrereozona antípoda es bastante espesa, alcanzando 1-1,5 $\mathrm{mm}$, y de ella se proyectan los septos hacia el interior del disepimentario (Lám. II, fig. 16).

\section{Discusión}

El espécimen descrito es muy similar a aquellos de $R$. cf. enorme y $R$. sp. A, también descritos en este trabajo. De $R$. cf. enorme se diferencia por la falta de espinas septales sobre los disepimentos de la región cardinal, por el alargamiento y mayor inclinación de los disepimentos de esta misma región en sección longitudinal, por la amplitud del tabulario y por la suave concavidad de las tabelas. De $R$. sp. A se distingue, esencialmente, por la mayor convexidad de la superficie cardinal, que proporciona un contorno subsemicircular, por la menor inclinación de los disepimentos de la región antípoda en el contacto con el tabulario y por la más suave concavidad de las tabelas. Puesto que disponemos de un solo ejemplar $\mathrm{y}$, por consiguiente, no hay posibilidad de analizar la variabilidad de los caracteres que definen la especie, consideramos más prudente mantener, por ahora, la asignación en nomenclatura abierta. 


\section{Distribución geográfica y estratigráfica}

Sección de Arpishmebulaq, SE de las Montañas de TianShan (prov. de Xinjiang, NO de China); Formación Arpishmebulaq (Unidad E), Devónico Inferior (Lochkoviense Superior).

\section{AGRADECIMIENTOS}

Este estudio ha sido realizado durante una estancia en régimen de sabático (SAB 1995-0410) del Prof. Liao Weihua en el Departamento de Geología de la Universidad de Oviedo, otorgada por la Subdirección General de Formación y Promoción del Conocimiento, del Ministerio de Educación y Cultura español. Expresamos también nuestro agradecimiento a los colegas del Instituto de Geología y Paleontología (Academia Sinica) de Nanjing, por su participación en la recogida de muestras, ayudando a uno de los autores (Liao, Weihua), a lo largo de una exploración científica en la provincia de Xinjiang (1988, 1992); asímismo, a los Srs. Song, del Instituto de Nanjing, y Joaquin Vázquez, del Departamento de Geología de Oviedo, por la realización de las láminas delgadas y fotografías, respectivamente, que ilustran este trabajo. Parte de las investigaciones realizadas han sido también subvencionadas por los proyectos PB 92-1008, PB 94-1324, otorgados por la DGICYT, y por otro proyecto de la Fundación Nacional de Ciencias Naturales de la República Popular de China, en el que participamos, entre otros, los autores de este trabajo.

\section{BIBLIOGRAFÍA}

Bayle, E. 1878. Fossiles principaux des terrains. Explication de la Carte Géologique de France, 4 (1), Láms. 1-158.

Besprozvannykh, N. I. 1968. Rugozy Tomchumyskikh sloev Salaira. In: Korally pograniychnykh sloev Silura $i$ Devona Altae-Sayanskoy gornoy oblasti $i$ Urala (Ed. A. B. Ivanoskiy). Nauka Moscow, 110-114 (en ruso).

Bulvanker, E. Z. 1958. Devonskie chetyrekhluchevye korally okrain Kuznetskogo basseyna. VSEGEI (Leningrad), 1-2, 1-212 (en ruso).

Cai, T. 1981. Subclass Rugosa. In: Atlas of Fossils of Northwest China, Xinjiang Volume, Part I (Early Palaeozoic). Regional Geological Survey Team and Geological Institute of Geological Bureau of Xinjiang, and the Geological Survey of Petroleum Bureau of Xinjiang. Geological Publishing House (Beijing), 21-39 (en chino).

Cai, T. \& Zeng, C. 1983. Subclass Rugosa. In: Atlas of Fossils of Northwest China, Xinjiang Volume, Part II (Late Palaeozoic). Regional Geological Survey Team and Geological Institute of Geological Bureau of Xinjiang, and Geological Survey of Petroleum Bureau of Xinjiang. Geological Publishing House (Beijing), 113704 (en chino).

Cao, X. 1975. Subclass Rugosa. In: Early Palaeozoic stratigraphy of western Dabashan Mountains (Ed. Y. Li, L. Song, Z. Zhou and J. Yang). Geological Publishing House (Beijing), 179-195 (en chino).
Cao, X. \& Ouyang, X. 1987. Rugose corals. In: The Late Silurian and Devonian Stratigraphy and Palaeontology in Luqu and Diebu districts of Western Qinling Mountains, Part I. Xian Institute of Geology and Mineral Resources, the Ministry of Geology and Mineral Resources and Nanjing Institute of Geology and Palaeontology, Academia Sinica. University Press (Nanjing), 139-202 (en chino, con resumen en inglés).

Etheridge, R. 1903. An unusually large form of Rhizophyllum, lately discovered in New South Wales. Records Geological Survey New South Wales, 7, 232233.

Ge, Z. \& Yü, Ch. 1974. Silurian corals. In: A handbook of the Stratigraphy and Palaeontology in Southwest China. Nanjing Institute of Geology and Palaeontology, Academia Sinica (Science Press), 165-173 (en chino).

Grabau, A. W. 1925. A summary of the faunas from the Sintan Shale. Bulletin Geological Survey of China, 7, 7785.

Guo, S. 1980. Subclass Rugosa. In: Atlas of Fossils of Northeast China, Part I (Palaeozoic). Shenyang Institute of Geology and Mineral Resources. Geological Publishing House (Beijing), 106-152 (en chino).

He, Y. 1978. Subclass Rugosa. In: Atlas of fossils of Southwest China, Sichuan Volume, Part I (Sinian to Devonian). Chengdu Institute of Geology and Mineral Resources. Geological Publishing House (Beijing), 98178 (en chino).

Hill, D. 1981. Rugosa and Tabulata. In: Treatise on Invertebrate Palaeontology, Part F, Coelenterata, Supplement I, F (S1) (Ed. R. A. Robinson). The Geological Society of America and the University of Kansas, 1-762.

Hill, D. \& Jell, J. S. 1969. On the rugose coral genera Rhizophyllum Lindström, Platyphyllum Lindström and Calceola Lamarck. Neues Jahrbuch GeologischePaläontologische Abhandlungen, 9, 534-551.

Ivanovskiy, A. B. 1965. Drevneyshie rugozy. Nauka Moscow, 1-152 (en ruso).

Koninck, L. G. de 1876-77. Recherches sur les fossiles paléozoiques de la Nouvelle-Galles du Sud (Australie). Memoir Geological Survey New South Wales (Paleontology), 6, 1-373.

Lavrusevich, A. I. 1971. Nekotorye rugozy iz pozdnesiluriyskikh i rannedevonskikh otlozheniy Tsentralnogo Tadzhikistana. Upravleniya Geologii Soveta Ministrov Tadzhikskoy SSR. Trudy Paleontologiya i Stratigrafiya, 4, 33-52, 123-135 (en ruso).

Li, S. \& Liao, W. 1994. Note on a new species of Rhizophyllum from Junggar. Acta Palaeontologica Sinica, 33, 246-248 (en chino, con resumen en inglés).

Liao, W. y Xia, F. 1996. On the geological age of the Arpishmebulaq Formation in eastern South Tianshan, Xinjiang. Xinjiang Petroleum Geology, 17 (2), 138-144 (en chino).

Lindström, G. 1883. Obersilurische Korallen von Tshau-tiën im nordöstlichen Teil der Provinz Sz-Tshwan. In: Beiträge zur Paläontologie von China (Ed. F. von Richthofen), 4, 50-74. 
Lyon, V. W. 1879. Descriptions of three new species of Calceolidae from the Upper Silurian rocks of Kentucky. Proceedings Academy Natural Sciences Philadelphia, 31, 43-46.

Norin, E. 1937. Geology of western Quruq Tagh, eastern Tian-Shan. In: Reports from the scientific Expedition to the Noth-western provinces of China under leadership of Dr. Sven Hedin, Sino-Swedish Expedition (Geology), III (1). Trychery Aktiebolaget Thule, 1-194.

Norin, E. 1941. Geologie reconnaissances in the chinese Tian-Shan. In: Reports from the scientific Expedition to the North-western provinces of China under leadership of Dr. Sven Hedin, Sino-Swedish Expedition (Geology), III (6). Trichery Aktiebolaget Thule, 1-229.

Oliver, W. A. Jr. 1964. New occurrences of the rugose coral Rhizophyllum in North America. Geological Survey Professional Papers, 475 D, 149-158.

Pocta, P. 1902. Anthozoaires et Alcyonaires. In: Sistème Silurien du centre de la Bohème (Ed. J. Barrande), 8 (2), 1-347.

Reed, F. R. C. 1924. Some new Ordovician and Silurian fossils. Geological Magazine, 61, 25-31.

Regnell, G. 1941. On the Siluro-Devonian fauna of Chöltagh, eastern Tian-Shan, Part I, Anthozoa. In: Reports from the scientific Expedition to the North-western provinces of China under leadership of Dr. Sven Hedin, Sino-Swedish Expedition (Geology), 5 (3). Trichery Aktiebolaget Thule, 1-64.

Römer, F. A. 1854. Erste Periode, Kohlen-Gebirge. Lethaea Geognostica, 1-788.

Römer, F. A. 1856. Bericht von einer geologischpaläontologischen Reise nach Schweden. Neues Jahrbuch Mineralogie Geognostica und Geologie, 794815.

Shearsby, A. J. 1905. On a new species of Rhizophyllum from the Upper Silurian rocks of Yass, New South Wales. Proceedings Linné Society New South Wales, 29, 86.9-870.
Shearsby, A. J. 1906. Notes on the operculate Madreporaria Rugosa from Yass, New South Wales. Geological Magazine, 3 (5), 547-552.

Soshkina, E. D. 1937. Korally verkhnego silura i nizhnego devona vostochnogo i zapadnogo sklonov Urala. Trudy paleozoologiskyi Institut, 6 (4), 1-153 (en ruso).

Soshkina, E. D. 1949. Devonskie korally Rugosa Urala. Trudy paleontologiskyi Institut, 15 (4), 1-162 (en ruso).

Soshkina, E. D. 1952. Opredelitel' devonskikh chetyrekhluchevykh korallov. Trudy paleontologiskyi Institut, 39, 1-178 (en ruso).

Spasskiy, N. Y. 1960. Devonskie chetyrekhluchevye korally Yuzhnogo Altaya i prilegayushchikh territoriy. Zapadnoi Leningrad gornoi Institut, 37 (2), 108-131 (en ruso).

Strusz, D. L. 1970. Rhizophyllum and Calceola from the Devonian of New South Wales. Department of National Development, Bureau of Mineral Resources, Geology and Geophysics. Palaeontological Papers, 108 (10), 281-302.

Sugiyama, T. 1940. Stratigraphical and palaeontological studies of the Gotlandian deposits of the Kitakami Mountainland. Science Reports Tohoku University, 21, 81-146.

Sutherland, P. K. 1965. Rugose corals of the Henryhouse Formation (Silurian) in Oklahoma. Bulletin Oklahoma Geological Survey, 109, 1-92.

Termier, H. et Termier, G. 1950. Paléontologie marocaine. Tome II: Invertebrés de l'Ere Primaire. Fasc. 1: Foraminifères, Spongiaires et Coelenterés. 1-218.

Wang, H. C. 1948. Note on a remarkable Rhizophyllum species from the Middle Silurian of Hueitze, Northern Yunnan. Palaeontological Novitiate, 2, 1-4.

Weyer, D. 1996. Calceolidae versus Goniophyllidae (Anthozoa, Rugosa; Silur-Devon). Abhandlungen und Berichte für Naturkunde Magdeburg, 19, 69-71.

Yoh, S. S. 1988. Devonian Calceolid corals of South China; the new classification and geological distribution. Professional Papers of Stratigraphy and Palaeontology, 21, 1-17 (en chino, con breve resumen en inglés).

Manuscrito recibido: 23 de enero, 1998 Manuscrito aceptado: 6 de abril, 1998 